\title{
Age-related changes in the morphology of tanycytes in the human female infundibular nucleus/median eminence
}

\author{
A. C. M. Koopman | M. Taziaux | J. Bakker (D)
}

GIGA Neurosciences, University of Liège, Liège, Belgium

\section{Correspondence}

Julie Bakker, GIGA Neurosciences, University of Liège, Liège, Belgium.

Email: jbakker@ulg.ac.be

Funding information

Nederlandse Organisatie voor

Wetenschappelijk Onderzoek, Grant/Award

Number: 453-08-003
Tanycytes are emerging as key players in the neuroendocrine control of gonadotrophinreleasing hormone $(\mathrm{GnRH})$ release. Rodent studies have demonstrated that the structural relationship between tanycytes and GnRH terminals in the median eminence is highly dynamic, regulated by gonadal steroids and undergoes age-related changes. The present study aimed to determine whether the number and organisation of tanycytes changes throughout life in the female infundibular nucleus/median eminence (INF/ME) region. Post-mortem hypothalamic tissues were collected at the Netherlands Brain Bank and were stained for vimentin by immunohistochemistry. Hypothalami of 22 control female subjects were categorised into three periods: infant/prepubertal, adult and elderly. We measured the fractional area covered by vimentin immunoreactivity in the INF. Qualitative analysis demonstrated a remarkable parallel organisation of vimentin-immunoreactive processes during the infant/prepubertal and adult periods. During the elderly period, this organisation was largely lost. Semi-quantitatively, the fractional area covered in vimentin immunoreactivity was significantly higher at the infant/prepubertal compared to the adult period and almost reached statistical significance compared to the elderly period. By contrast, the number of tanycyte cell bodies did not appear to change throughout life. The results of the present study thus demonstrate that the number and structure of tanycytic processes are altered during ageing, suggesting that tanycytes might be involved in the age-related changes observed in $\mathrm{GnRH}$ release.

\section{KEYWORDS}

ageing, human, infundibular nucleus, median eminence, tanycytes

\section{1 | INTRODUCTION}

Tanycytes, which are specialised bipolar glial cells, are emerging as key players in the neuroendocrine control of gonadotrophin-releasing hormone $(\mathrm{GnRH})$ secretion in vertebrates. ${ }^{1-5}$ In rodents, four subsets of tanycytes have been identified. Of these, the $\beta 1$ type was shown to be closely related to $\mathrm{GnRH}$ release. ${ }^{4}$ These tanycytes line the floor and wall of the third ventricle, and extend their processes to the portal vasculature of the median eminence (ME), ${ }^{4}$ where they physically interact with $\mathrm{GnRH}$ terminals through apposition to $\mathrm{GnRH}$ fibres. This structural relationship is highly dynamic and regulated by gonadal steroids throughout the reproductive cycle. Tanycytes can influence the secretion of GnRH from axon terminals to the portal capillaries by two different means. The first involves cell-to-cell communication via signalling molecules released by tanycytes, which modulate the activity of $\mathrm{GnRH}$ neurones. ${ }^{4}$ The second mechanism, sometimes called the remodelling theory, is exemplary of the plastic nature of tanycytes. Specifically, rodent data showed that during most of the ovarian cycle, $\mathrm{GnRH}$ synaptic terminals are retracted and completely enwrapped by tanycyte end-feet, preventing the release of $\mathrm{GnRH}$ into the hypophyseal-portal circulation. ${ }^{1-6}$ By contrast, during the pro-oestrous phase of the cycle (when the GnRH surge occurs), these end-feet are retracted, thereby exposing $\mathrm{GnRH}$ synaptic terminals and allowing them to make contact with the hypophyseal-portal blood. ${ }^{5}$ Indeed, 
TABLE 1 Clinicopathological data

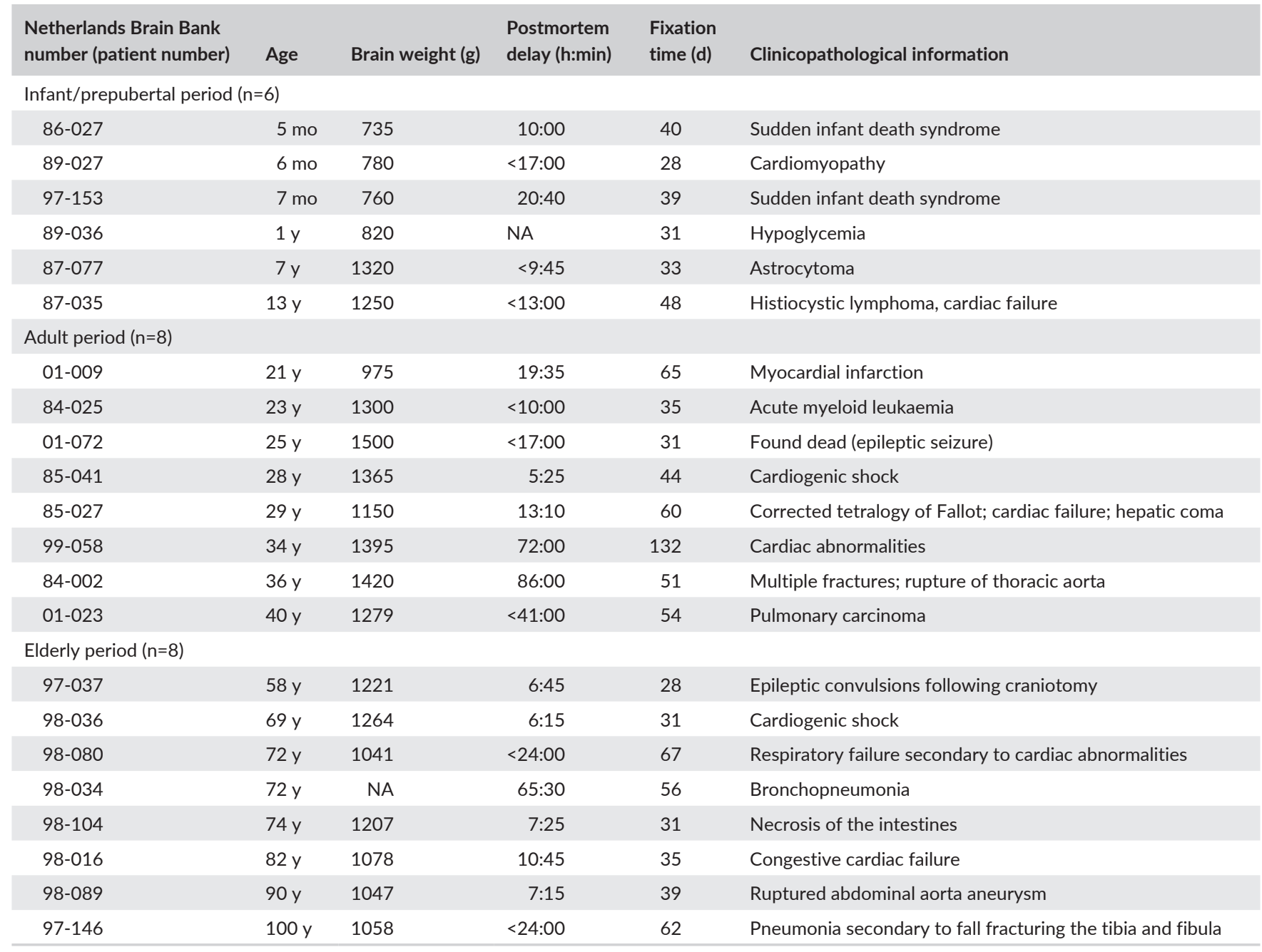

NA, not available.

chemical removal of tanycytes in rats prevents the occurrence of a surge in luteinising hormone and consequently ovulation. ${ }^{7}$ Similarly, when semaphorin7A (a protein expressed by tanycytes) receptors were genetically invalidated in mice, neuronal and glial arrangements in the ME were changed, suppressing the normal oestrous cycle and consequently fertility. ${ }^{6}$ Furthermore, it has been shown that tanycytes undergo morphological changes during ageing, a physiological condition often characterised by a loss of oestrogen feedback on hypothalamic GnRH neurones and a general decline in the sensitivity of $\mathrm{GnRH}$ neurones to oestrogens. ${ }^{8,9}$ For example, ageing has long been associated with a progressive decline in the number of tanycytes, ${ }^{10}$ as well as some morphological changes, in rats. ${ }^{11}$ More specifically, in young (4-5 months old) rats, tanycytes are long, narrow and neatly lined up. With increasing age (ie, 22-24 months old), tanycyte processes lose this organisation in the pericapillary zone and become thicker. ${ }^{11}$ These findings strongly suggest that $\mathrm{GnRH}$ release in the ageing rat is altered by structural organisational changes. However, whether tanycytes have a similar function in humans and whether human menopause is associated with similar structural and morphological changes is unknown. Therefore, in the present study, using post-mortem brain tissues and immunohistochemical staining against vimentin (a marker of tanycytes), we analysed the distribution of tanycyte cell bodies and processes in the female human infundibular and median eminence (INF/ME) region. In addition, we determined whether the number and organisation of tanycytes at the level of the INF/ME changes throughout life in the female human hypothalamus.

\section{2 | MATERIALS AND METHODS}

\section{1 | Human brain tissue}

The hypothalami of 22 female subjects (Table 1) were obtained from The Netherlands Brain Bank. These subjects had given permission for brain autopsy and the use of their medical information and tissue for research projects. The subjects were categorised into three groups: infant/prepubertal (5 months to 13 years), adult (21-40 years) and elderly (58-100 years). Exclusion criteria for the subjects were a history of endocrine dysregulation (ovarian, uterine or breast cancer; recent abortion; recent pregnancy; acquired immunodeficiency syndrome; anorexia), the use of corticosteroids or drugs affecting 


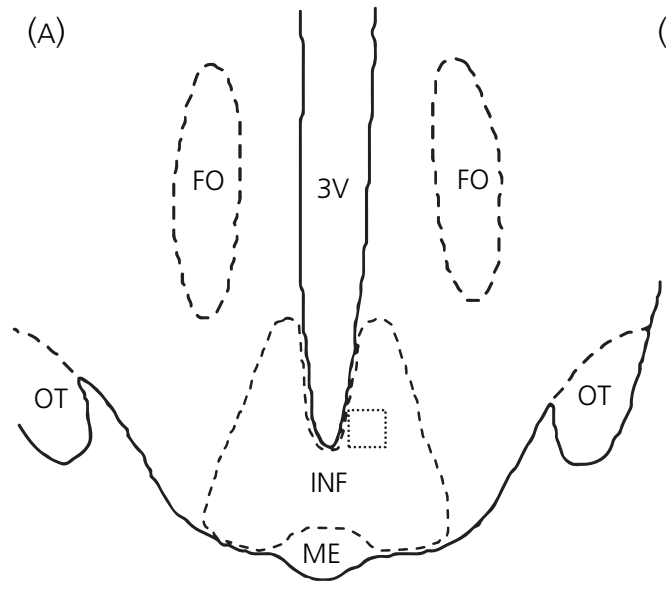

(B)

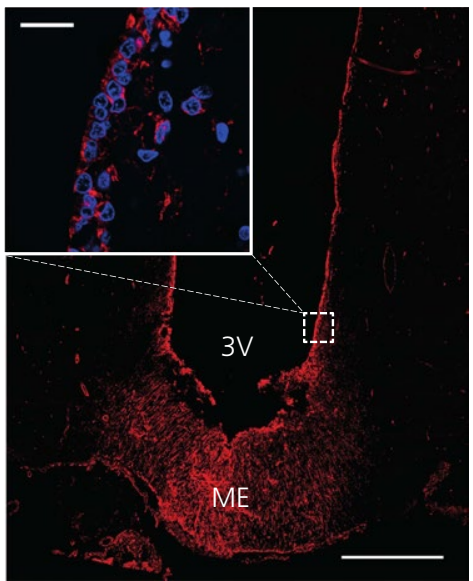

FIGURE 1 (A) Schematic representation of the localisation of images taken to quantify vimentin immunoreactivity. Dashed lines demarcate anatomical structures, whereas the dotted lined box represents the approximate area at which a digital image of vimentin immunoreactivity was acquired. (B) Localisation of tanycyte cell bodies (orange: vimentin-immunoreactive; blue: 4',6-diamidino-2-phenylindole) in a representative patient (25 years; NBB 01-072). The dashed box surrounds the area magnified in the panel. Scale bars $=1 \mathrm{~mm}$ and $20 \mu \mathrm{m}$ in the image and panel, respectively. 3V, third ventricle; FO, fornix; INF, infundibular nucleus; ME, median eminence; OT, optic tract

the hypothalamic-pituitary-gonadal (HPG) axis during at least the last 3 months before death, and neurodegenerative or psychiatric diseases.

\section{2 | Histology}

Hypothalami were formalin-fixed, paraffin-embedded and cut serially in $6-\mu \mathrm{m}$ coronal sections from rostral to caudal. For orientation purposes, every 100th section was collected on a SuperFrost/Plus (Menzel, Braunschweig, Germany) slide and stained with $0.5 \%$ thionin. Every 50th section was then collected from the rostral start of the ME until the appearance of the mammillary bodies and stained for vimentin by immunohistochemistry (see below). Vimentin is an intermediate filament present in all tanycyte types, ${ }^{4}$ and is important in cell migration and maintaining cell integrity. ${ }^{12}$ Sections were deparaffinised and rehydrated through xylene and decreasing grades of ethanol. Unless stated otherwise, all incubations were carried out at room temperature and all washes were performed using Tris-buffered saline (TBS; $0.05 \mathrm{~mol} \mathrm{~L}^{-1}$ Tris and $0.9 \% \mathrm{NaCl}$; $\mathrm{pH}$ 7.6).

\section{3 | Vimentin immunohistochemistry}

Sections were first processed for antigen retrieval by placing them in citrate buffer $\left(0.1 \mathrm{~mol} \mathrm{~L}^{-1}\right.$ citric acid, $0.1 \mathrm{~mol} \mathrm{~L}^{-1}$ trisodium citrate, $\mathrm{pH}$ 6.0) in a microwave ( 5 minutes, $700 \mathrm{~W}$ ). Next, sections were incubated sequentially in $5 \%$ normal horse serum (NHS; 1 hour) in TBS-0.5\% Triton- $\mathrm{X} 100$ (TBST) and overnight at $4{ }^{\circ} \mathrm{C}$ with a mouse monoclonal antibody against vimentin (dilution 1:1000; M 0725; Dako Cytomation, Glostrup, Denmark), diluted in 5\% NHS-TBST. They were then incubated for 1 hour in biotinylated horse anti-mouse antibody (dilution 1:400 in 5\% NHS-TBST; Vector, Bulrlingame, CA, USA) followed by 1 hour in a streptavidin Cy3 antibody (dilution 1:400 in TBST). To counteract autofluorescence, the sections were immersed in a solution of $0.03 \%$ Sudan Black B (Merck, Darmstadt, Germany) dissolved in $70 \%$ ethanol for 7 minutes. The slides were then coverslipped with Vectashield (Vector) and stored at $4^{\circ} \mathrm{C}$ until use.

\section{4 | Vimentin antibody specificity}

The monoclonal mouse anti-vimentin antibody was used successfully in previous immunohistochemical experiments on hypothalamic sections from humans. ${ }^{13}$ The specificity has been demonstrated previously. $^{14}$

\section{5 | Semi-quantification of vimentin immunoreactivity}

All sections of each subject were carefully examined by an investigator (AK) who was blind to the nature of the patients. Two sections containing the majority of vimentin-immunoreactive processes in the INF, at the location theoretically corresponding to the location of $\beta 1$ tanycytes (Figure 1), ${ }^{4}$ were selected for each subject. Digital images were acquired using a $40 \times$ objective (Plan-NEOFLUAR lens) on an Imager.Z1 microscope (Carl Zeiss, Oberkochen, Germany) mounted with a Retiga EXi Fast 1294 camera (Qimaging, Surrey, BC, Canada) and connected to FLuo'up image analysis software (Explora Nova, La Rochelle, France). Using computer-assisted image analysis (ImageJ, version 1.48v; National Institutes of Health, Bethesda, MD, USA), images were made binary and a manual threshold was used to distinguish the labelled material (tanycyte processes) from the background. Pixels in the image whose values lay within the range of this threshold were converted to black; pixels with values outside this range were converted to white. The fractional area covered by vimentin immunoreactivity was measured unilaterally in two sections and averaged. The fractional area covered by vimentin immunoreactivity was defined as the surface (expressed in pixels) covered by immunoreactive vimentin processes divided by the total area (ie, the entire image; $1392 \times 1040$ pixels) multiplied by 100 . 

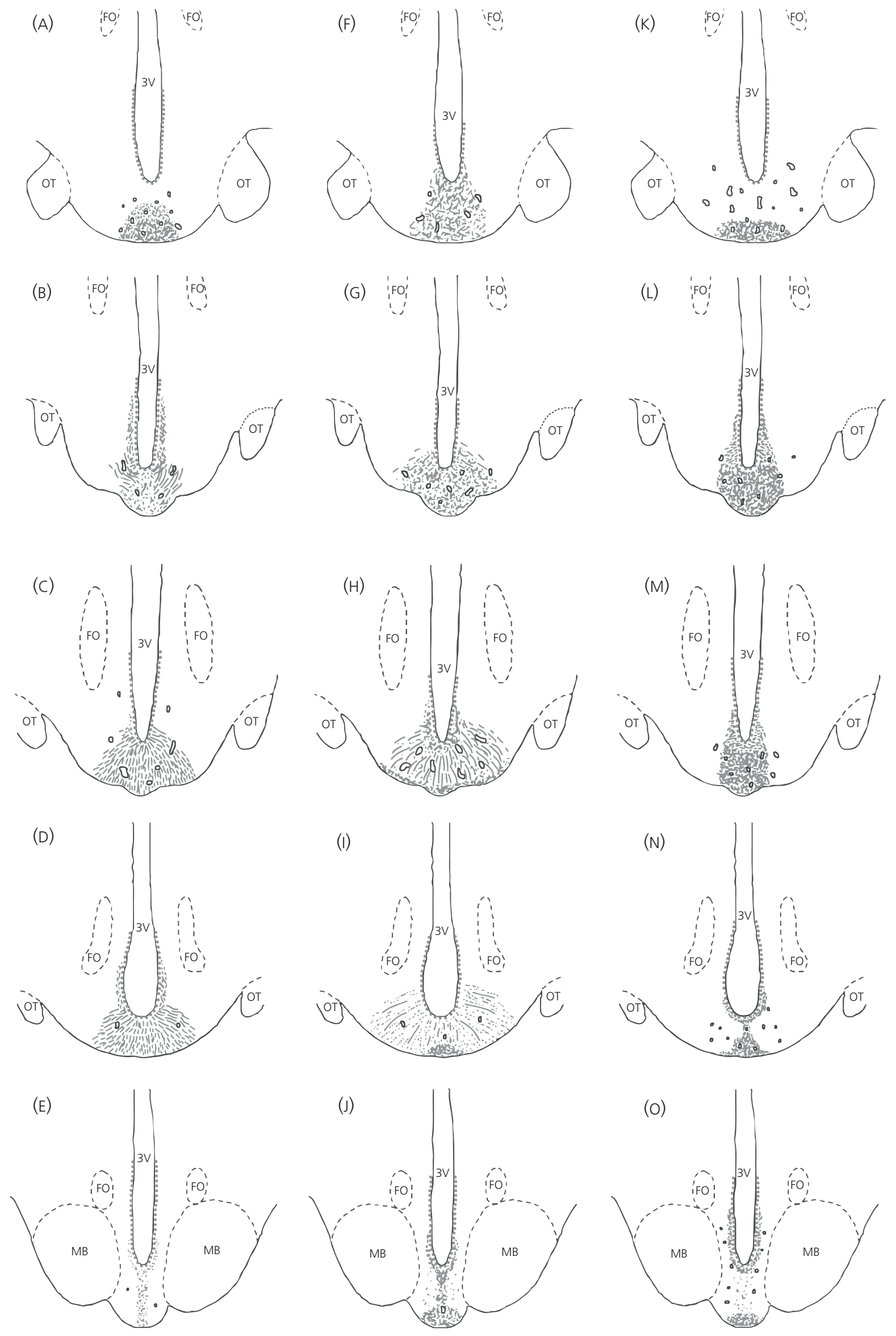
FIGURE 2 Schematic drawing from anterior to posterior in three representative female hypothalami from different stages of life (A-E: 6 months, NBB 89-027; F-J: 40 years, NBB 01-023; K-O: 72 years, NBB 98-080), to illustrate the distribution of vimentin-immunoreactive cell bodies and processes. Vimentin-immunoreactive cells are represented by light-grey closed circles around the border of the third ventricle, whereas vimentin-immunoreactive fibers are represented by light-grey lines. The dark-grey shapes represent capillaries. 3V, third ventricle; FO, fornix; MB, mammillary body; OT, optic tract

\section{6 | Quantification of tanycyte cell bodies}

The same two sections per subject used for the semi-quantification of vimentin immunoreactivity were analysed again to determine the position where a relatively clear and organised row of tanycyte cell bodies could be observed (for an example, see Figure 1B; for a schematic representation, see Figure 2). Digital images at this position were then acquired using a $63 \times$ objective (Plan-NEOFLUAR lens) with the same microscope and camera reported above, connected to MERCATOR image analysis software (Explora Nova). The cell bodies were manually counted unilaterally in two sections and these values were averaged for each subject.

\subsection{Statistical analysis}

Data were tested for the assumptions of normality and homogeneity of variances by looking at the histogram and scatterplot, respectively, and double-checked with a Kolmogorov-Smirnov and Levene's test, respectively. The data met the assumption of normality, whereas the $P$-value of the Levene test was borderline $(P=.050)$ for the fractional area covered by vimentin immunoreactivity. One-way ANOVAs with the stage of life (infant/prepubertal vs adult vs elderly) as independent factor were used to analyse the overall difference in vimentin immunoreactivity in the INF and the overall difference in number of tanycyte cell bodies. A general linear model was used to determine the effects of possible confounding variables such as post-mortem delay and fixation time. Linear regression analysis was carried out to examine and visualise the relationship between age and vimentin immunoreactivity. Because of the borderline heteroscedasticity, ANOVAs were followed by a Games-Howell post-hoc test where appropriate. $P<.05$ was considered statistically significant.

\section{3 | RESULTS}

Vimentin expression in the INF/ME region was very similar to that reported by previous studies in different species. ${ }^{4}$ Vimentinimmunoreactive cell bodies are strongly expressed in the ventral and lateral walls of the third ventricle, between the rostral and caudal borders of the ME. Along the walls of the ventricle, they line up in a relatively organised row. In many cases, this row contained more than one layer of cells. These cells were mainly round, oval or pear-shaped and showed an onset of processes (for an example, see Figure 1B). Tanycytes processes either reached the basal brain surface or terminated in the ME. In all age groups, the distribution of vimentin immunoreactivity follows similar rostro-caudal topography, with the majority of vimentin-immunoreactive processes being found in the rostral to middle part of the INF/ME region (Figure 2).

Systematic examination of vimentin immunoreactivityin the INF/ME region yielded obvious qualitative and semi-quantitative age-related differences. At a morphological level, we observed a marked difference in vimentin-immunoreactive processes at different stages of life. Namely, in the infant/prepubertal and adult groups, tanycyte processes showed remarkable organisation: they mostly ran parallel to each other in an arch-like manner from the ventricular wall to the ME and hardly ever crossed (Figure 3A,B). Moreover, tanycyte
FIGURE 3 Qualitative difference in the organisation of tanycyte processes in hypothalami of three representative females from different stages of life (1 year, NBB 89-036: A; 23 years, NBB 84-025: B; 100 years, NBB 97-146: C). The dashed boxes illustrate the areas magnified in the panels. Scale bars $=1 \mathrm{~mm}$ in the large images, $50 \mu \mathrm{m}$ in the panels. $3 \mathrm{~V}$, third ventricle; $\mathrm{ME}$, median eminence
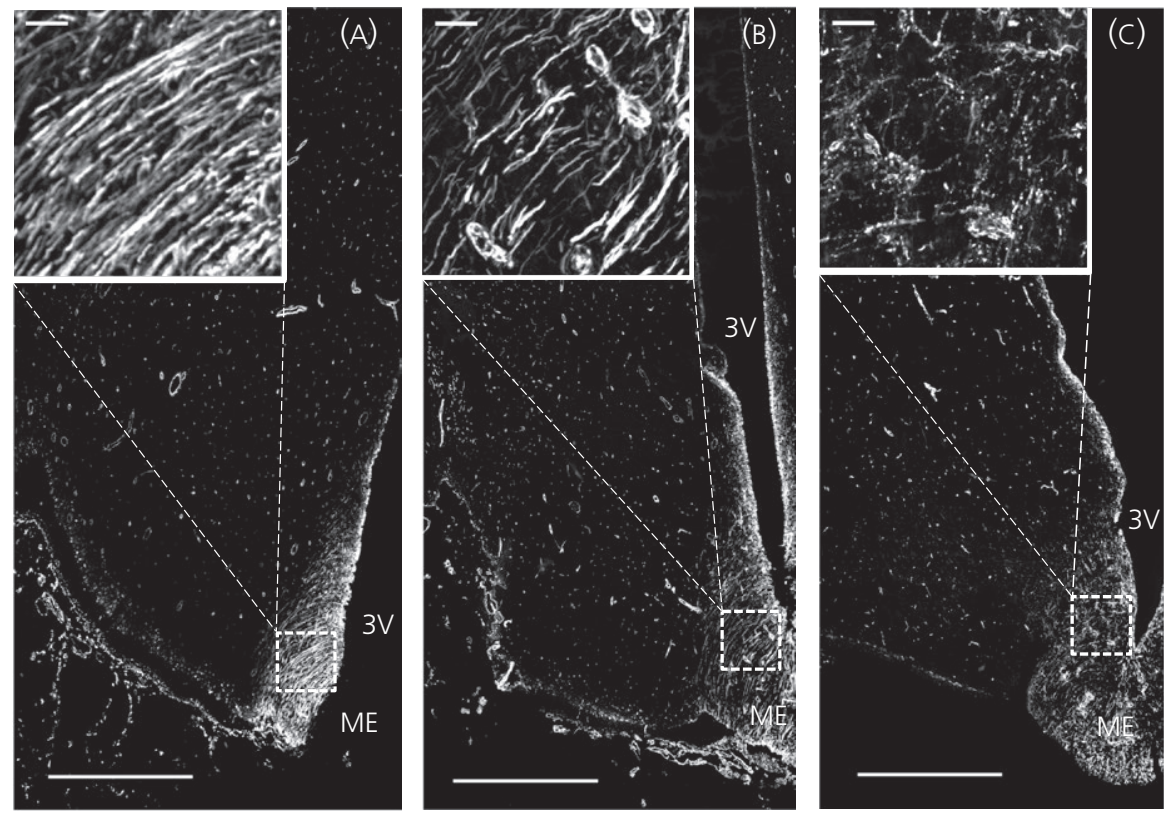
processes in these groups tended to be longer compared to those in the elderly group, meaning that they continued on uninterrupted for a relatively long distance. In the elderly group, on the other hand, the processes lost their organisation, crossed often, and were generally shorter (Figure 3C). This difference is also represented schematically in Figure 2.

A subsequent semi-quantitative analysis was performed to assess whether the fractional area covered by vimentin immunoreactivity changes over a lifetime. Overall, higher vimentin immunoreactivity was found in the infant/prepubertal group compared to the adult and the elderly groups (Figure 4A). A one-way ANOVA revealed a significant effect of the stage of life $\left(F_{2,19}=9.309 ; P=.002\right)$. A subsequent Games-Howell post-hoc test showed that vimentin immunoreactivity was significantly higher in the infant/prepubertal group compared to the adult group $(P=.028)$. The difference between the infant/prepubertal and the elderly groups almost reached statistical significance $(P=.064)$. No significant difference in vimentin immunoreactivity was found between the adult and elderly groups $(P=.581)$.

A linear regression with age as the independent factor and fractional area covered by vimentin immunoreactivity as the dependent variable showed a negative (albeit not statistically significant) correlation ( $r=.403$; $P=.063 ; n=22$ ) (Figure 4B). Age accounted for approximately $16 \%$ of the variance in vimentin immunoreactivity $\left(r^{2}=.162 ; F_{1,20}=3.871\right)$.

Tanycyte cell bodies were then examined to determine whether they also differed across the lifespan. A one-way ANOVA on the number of tanycyte cell bodies did not show a significant effect of the stage of life $\left(F_{2,19}=0.160 ; P=.853\right)$ (Figure $\left.5 \mathrm{~A}\right)$. A linear regression with age as the independent factor and average number of tanycyte cell bodies as the dependent variable showed a nonsignificant positive correlation $(r=.094 ; P=.676 ; n=22)$ (Figure $5 B)$, with an $r^{2}$ of $.009\left(F_{1,20}=0.180\right)$. Careful examination of vimentin-immunoreactive cell bodies throughout the rostro-caudal extent of the INF/ME region revealed strong inter- and intrapersonal differences in cell body abundance, diameter and shape, although there were no apparent group differences.

\section{1 | Confounding factors}

No significant effects of post-mortem delay or fixation time on vimentin immunoreactivity were observed (all $P>$.38).

\section{4 | DISCUSSION}

In the present study, we showed for the first time in humans that tanycytes undergo age-related morphological modifications in the female INF/ME. Specifically, in the infant/prepubertal and adult periods, tanycytes send long, linearly structured processes from the ventricular wall to the ME in an arch-like manner. With age, tanycyte processes markedly lose their organisation. Furthermore, we showed that vimentin immunoreactivity in the INF is greater during the infant/ prepubertal compared to the adult period. These findings suggest that the access of GnRH to the portal capillary system might be age- and steroid-dependent in women.

\section{1 | Distribution of vimentin immunoreactivity in the human hypothalamus}

We observed that fibres and cells expressing vimentin immunoreactivity were predominantly found in the rostral to middle part of the INF/ME region in all age groups. This is in agreement with the distribution of tanycytes shown in rats. ${ }^{15}$ Tanycytes in the arcuate nucleus (the rodent homologue of the human INF) have also been described in the mouse, ${ }^{16-18}$ hamster ${ }^{19}$ and monkey. ${ }^{20}$ The INF/ME region is also reported as the predominant site for tanycytes in a previous study in humans, although the study used only elderly subjects and did not elaborate upon the rostrocaudal distribution of tanycyte processes. ${ }^{13}$ The distribution of tanycyte cell bodies also matches earlier descriptions. $^{21}$

\subsection{Qualitative stage of life difference in tanycyte processes}

A thorough examination of the tanycyte processes at all stages of life revealed age-related differences in organisation. Namely, in the infant/prepubertal and adult groups, the tanycytes that spanned the INF/ME region were generally well-organised, ran parallel to each other, seldom crossed and often covered a relatively large distance. By contrast, in the elderly group, tanycyte processes often crossed and rarely showed a parallel organisation, leading to an overall marked disorganisation. Tanycytes in the elderly group also appeared to span a shorter distance, although their crossing and/or the plane of the sections could contribute to this. These findings are consistent with earlier studies conducted in rodents. Yin and Gore ${ }^{9}$ reported a loss of perpendicular orientation with age, and a disorganisation of both the tanycyte processes and their relationship with the surrounding structures of the pericapillary zone. ${ }^{10}$ Zoli et al. ${ }^{10}$ also noted that the processes of tanycytes are more tortuous in old compared to young rats. The causes and consequences of this disorganisation remain speculative, although, given the importance of tanycyte functionality in neuroendocrine control and specifically in effective $\mathrm{GnRH}$ release, tanycyte disorganisation likely alters $\mathrm{GnRH}$ release into the portal system. Several studies have addressed the question of what happens to $\mathrm{GnRH}$ after menopause, despite the fact that the measurement of hypothalamic $\mathrm{GnRH}$ secretion is notoriously difficult because it is not accurately reflected by $\mathrm{GnRH}$ levels in peripheral blood. ${ }^{22}$ However, human studies using indirect methods suggest an overall increase in the total amount of $\mathrm{GnRH}$ being released and also that this continues to increase during ageing. ${ }^{23,24}$ This is consistent with findings of increased $\mathrm{GnRH}$ gene expression in postmenopausal women. ${ }^{25}$ Although the removal of oestrogen-negative feedback that accompanies menopause likely plays a large role in this initial increase observed in $\mathrm{GnRH}$ release, it is not known why the total amount of $\mathrm{GnRH}$ being released would continue to increase during ageing. The latter suggests that another mechanism could play a role as well, such as a decreased functionality of tanycytes. The question remains as to whether the observed changes occur as a result of ageing or menopause, although studies comparing young and old postmenopausal women indicate 

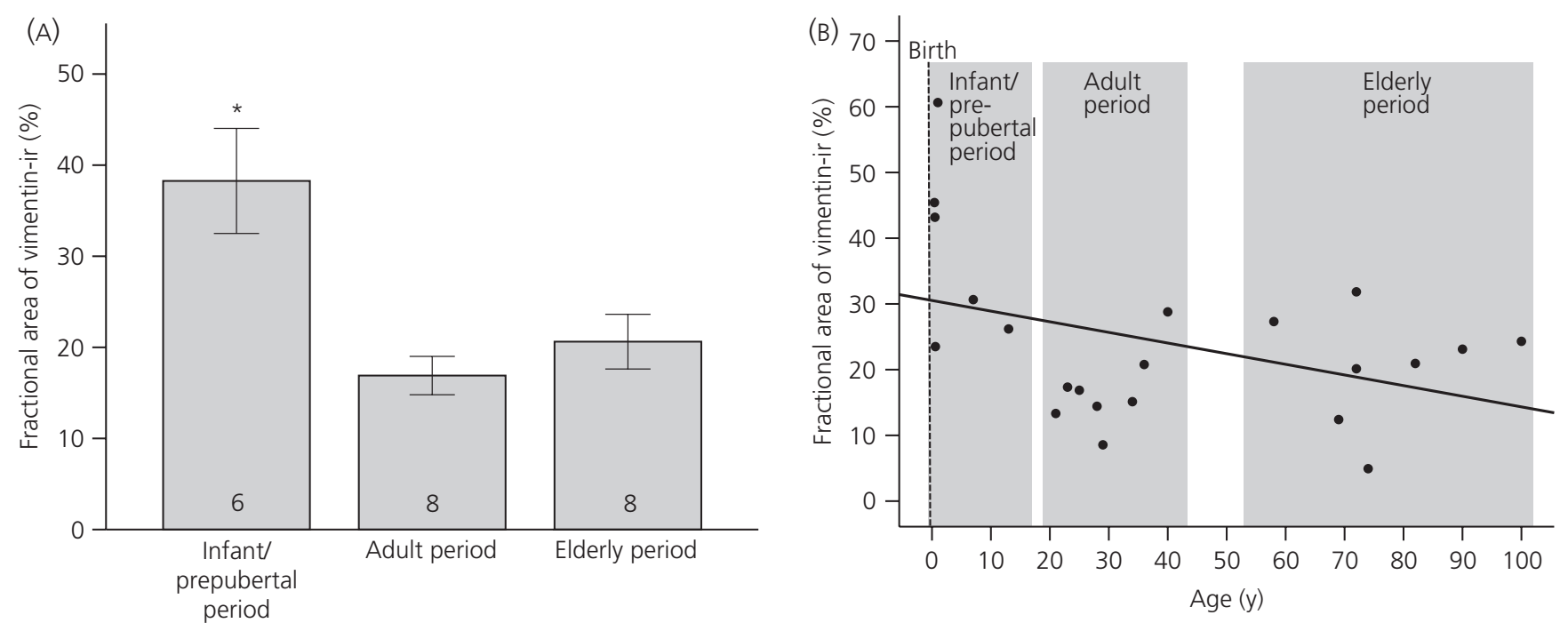

FIGURE 4 (A) Fractional area (percentage of surface) covered by vimentin immunoreactivity (vimentin-ir) per stage of life. ${ }^{*} P<.05$ vs the adult period. Error bars reflect the mean \pm SEM. (B) Correlation between the fractional area covered by vimentin immunoreactivity and age (from 5 months old to 100 years old). The line represents the regression line
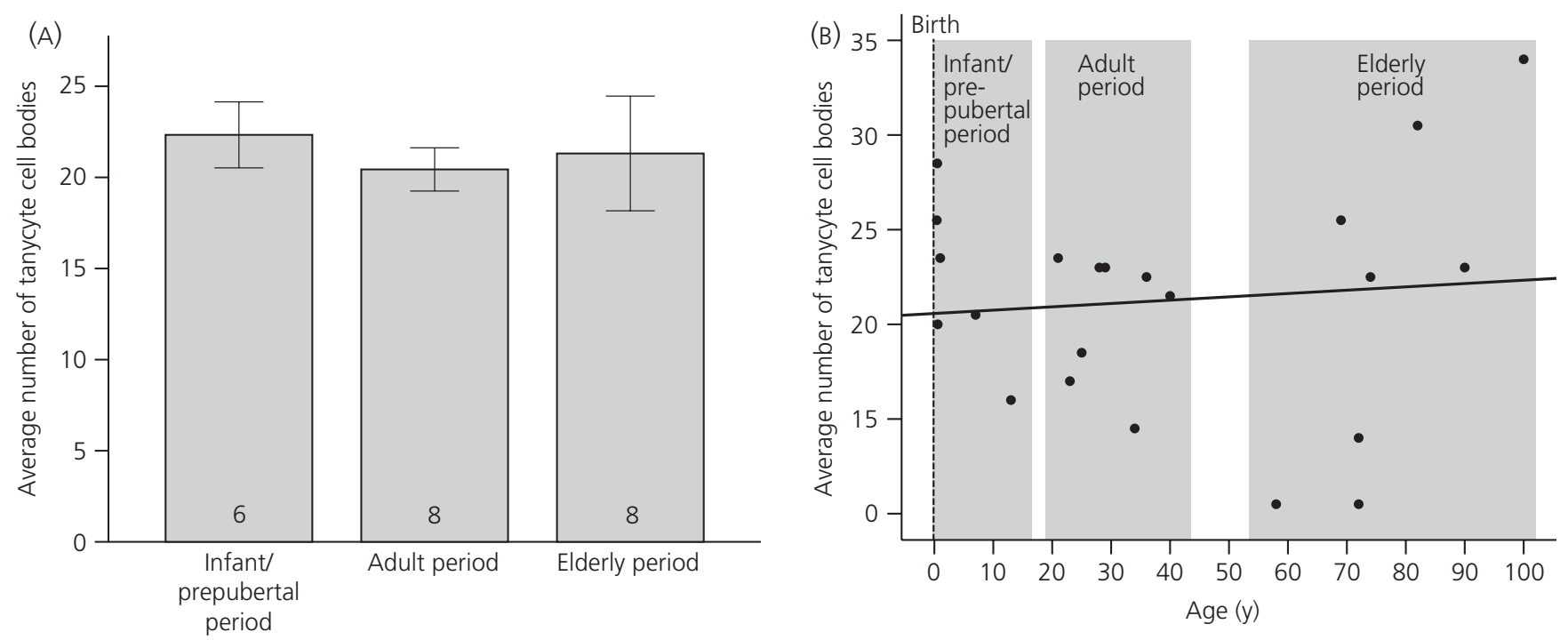

FIGURE 5 (A) Average number of tanycyte cell bodies counted per stage of life. Error bars reflect the mean \pm SEM. (B) Correlation between the average number of tanycyte cell bodies counted and age (from 5 months old to 100 years old). The line represents the regression line

that the neuroendocrine system undergoes age-related alterations that are independent of those occurring during menopause but may contribute to reproductive senescence. ${ }^{24}$

\subsection{Semi-quantitative stage of life difference in vimentin immunoreactivity}

Besides these age-related morphological changes in tanycyte processes, we also found that the amount of vimentin immunoreactivity was significantly higher in the infant/prepubertal compared to the adult period, with a nonsignificant difference compared to the elderly period. Previous research using alternative markers of tanycytes (dopamine and cyclic AMP-regulated phosphoprotein mr 32 [DARPP-32] and glial fibrillary acidic protein) identified a progressive loss of tanycytes with age in rats, although it was not reported whether this difference is significant between the adult and elderly stages. ${ }^{10}$ In the present study, no differences were found between the adult and elderly periods, although we were only able to assess the fractional area covered by vimentin immunoreactivity. We showed that vimentin immunoreactivity in the elderly subjects covered approximately the same fractional area as in the adult group, notwithstanding the fact that it is very differently organised. Unfortunately, it is this same disorganisation that prevented us from counting and comparing the number of tanycyte processes. The lack of difference between the adult and elderly groups might further be explained by the use of vimentin as a marker of tanycytes because its expression has been 
shown to increase with ageing in the entorhinal cortex. ${ }^{26,27}$ However, none of these studies reported any particular disorganisation such as that observed in the present study in the ME.

The higher amount of vimentin immunoreactivity observed at the infant/prepubertal period might reflect a period of neuromorphogenesis because vimentin is an intermediate filament protein that has been shown to be involved in cell migration. ${ }^{12}$ However, this is rather unlikely given that hypothalamic maturation occurs primarily during intrauterine life in humans. ${ }^{28} \mathrm{~A}$ more likely explanation is that the high fractional area covered by vimentin immunoreactivity in the infant/prepubertal period actually reflects a large amount of tanycyte processes enwrapping the GnRH nerve terminals to silence $\mathrm{GnRH}$ release. The HPG axis is generally silenced during the infant/prepubertal period, with the exception of high activity during the first 3 months after birth, a period known as mini-puberty. ${ }^{29}$ Given that one of the functions of tanycytes is to enwrap GnRH nerve terminals, the high fractional area covered by vimentin immunoreactivity could partly explain the absence of $\mathrm{GnRH}$ release during the majority of childhood. A next step might be to investigate the expression of semaphorin7A at different stages of life because this protein has recently been shown to be crucial for tanycyte plasticity. ${ }^{6}$ Interestingly, several growth factors involved in the initiation of puberty (such as TGF $\alpha$ and TGF $\beta 1$ ) have been shown to engage GnRH neurones through tanycytes, ${ }^{6,30-32}$ which suggests a common upstream signal that affects the tanycyte$\mathrm{GnRH}$ relationship and the start of puberty.

\subsection{Tanycyte cell bodies at different stages of life}

No significant differences were found in the number of tanycyte cell bodies at different stages of life. Moreover, no differences in cell size or shape were found either, in contrast to what is suggested by rat data. ${ }^{33}$ In young ( 3 weeks old) compared to adult ( 24 weeks old) rats, for example, tanycyte cell bodies were found to be smaller and more regular in outline. It appears that the situation in humans is more complex and less clear than in rodents, with large inter- and intrapersonal differences, although there were no overall group differences in our sample. It is worth noting that rodent studies showing a decrease in the number of tanycytes with increased age only analysed tanycyte processes ${ }^{10}$ or the amount of appositions with GnRH terminal membranes ${ }^{11}$ and not tanycyte cell bodies. In other words, what happens to the number of tanycyte cell bodies during ageing in rodents is also unclear. Our results might suggest that the number, shape and size of tanycyte cell bodies do not change from birth to old age in humans. However, it should be noted that our sample size (the number of cells counted per subject) was quite small, and the integrity of the tissue next to the third ventricle (where the cell bodies are located) was not optimal in some subjects. Thus, some caution is warranted.

To conclude, the results of the present study show that the organisation of tanycytic processes in the INF/ME region is altered during ageing, thereby suggesting that they might be involved in the age-related changes observed in $\mathrm{GnRH}$ release. Besides their role in $\mathrm{GnRH}$ regulation, hypothalamic tanycytes are now being studied as neural stem cells, ${ }^{34}$ in relation to feeding and energy balance, ${ }^{35}$ and more functions are just beginning to be explored. ${ }^{4,36}$ Alterations in tanycyte morphology and organisation with age could thus have consequences that go far beyond the HPG axis.

\section{ACKNOWLEDGEMENTS}

This research was supported by a $\mathrm{VICl}$ grant of the Nederlandse Organisatie voor Wetenschappelijk Onderzoek (NWO; 453-08-003) to JB. We thank A. Sluiter, R. Balesar, B. Fisser and J. J. van Heerikhuize for their technical assistance. Brain material was provided by the Netherlands Brain Bank (coordinator: Dr I. Huitinga). J. Bakker is a research director at the Fonds National de la Recherche Scientifique (FNRS). M. Taziaux was a postdoctoral researcher at the FNRS.

\section{DISCLOSURE}

The authors have nothing to disclose.

\section{REFERENCES}

1. Silverman RC, Gibson MJ, Silverman A-J. Relationship of glia to GnRH axonal outgrowth from third ventricular grafts in hpg hosts. Exp Neurol. 1991;114:259-274.

2. King JC, Letourneau RJ. Luteinizing hormone-releasing hormone terminals in the median eminence of rats undergo dramatic changes after gonadectomy, as revealed by electron microscopic image analysis. Endocrinology. 1994;134:1340-1351.

3. Prevot V, Croix D, Bouret S, et al. Definitive evidence for the existence of morphological plasticity in the external zone of the median eminence during the rat estrous cycle: implication of neuro-glioendothelial interactions in gonadotropin-releasing hormone release. Neuroscience. 1999;94:809-819.

4. Rodríguez EM, Blázquez JL, Pastor FE, et al. Hypothalamic tanycytes: a key component of brain-endocrine interaction. Int Rev Cytol. 2005;247:89-164.

5. Prevot V, Bellefontaine N, Baroncini M, et al. Gonadotrophin-releasing hormone nerve terminals, tanycytes and neurohaemal junction remodelling in the adult median eminence: functional consequences for reproduction and dynamic role of vascular endothelial cells. J Neuroendocrinol. 2010;22:639-649.

6. Parkash J, Messina A, Langlet F, et al. Semaphorin7A regulates neuroglial plasticity in the adult hypothalamic median eminence. Nat Commun. 2015;6:6385.

7. Rodríguez EM, Peña P, Aguado LI, Schoebitz K. Involvement of tanycytes in the control of gonadotropin secretion. In: Lofts B, Holmes WN, eds. Current Trends in Comparative Endocrinology. Hong Kong: Hong Kong University Press; 1985:113-116.

8. Yin W, Gore AC. Neuroendocrine control of reproductive aging: roles of GnRH neurons. Reproduction. 2006;131:403-414.

9. Yin W, Gore AC. The hypothalamic median eminence and its role in reproductive aging. Ann NY Acad Sci. 2010;1204:113-122.

10. Zoli M, Ferraguti F, Frasoldati A, Biagini G, Agnati LF. Age-related alterations in tanycytes of the mediobasal hypothalamus of the male rat. Neurobiol Aging. 1995;16:77-83.

11. Yin W, Mendenhall JM, Monita M, Gore AC. Three-dimensional properties of GnRH neuroterminals in the median eminence of young and old rats. J Comp Neurol. 2009;517:284-295.

12. Herrmann H, Bär H, Kreplak L, Strelkov SV, Aebi U. Intermediate filaments: from cell architecture to nanomechanics. Nat Rev Mol Cell Biol. 2007;8:562-573

13. Baroncini M, Allet C, Leroy D, Beauvillain JC, Francke JP, Prevot V. Morphological evidence for direct interaction between 
gonadotrophin-releasing hormone neurones and astroglial cells in the human hypothalamus. J Neuroendocrinol. 2007;19:691-702.

14. Osborn M, Debus E, Weber K. Monoclonal antibodies specific for vimentin. Eur J Cell Biol. 1984;34:137-143.

15. Rodríguez EM, González CB, Delannoy L. Cellular organization of the lateral and postinfundibular regions of the median eminence in the rat. Cell Tissue Res. 1979;201:377-408.

16. Millhouse OE. A Golgi study of third ventricle tanycytes in the adult rodent brain. Z Zellforsch Mikrosk Anat. 1971;121:1-13.

17. Bascó E, Woodhams PL, Hajós F, Balázs R. Immunocytochemical demonstration of glial fibrillary acidic protein in mouse tanycytes. Anat Embryol (Berl). 1981;162:217-222.

18. Mullier A, Bouret SG, Prevot V, Dehouck B. Differential distribution of tight junction proteins suggests a role for tanycytes in bloodhypothalamus barrier regulation in the adult mouse brain. J Comp Neurol. 2010;518:943-962.

19. Barrett P, Ivanova E, Graham ES, et al. Photoperiodic regulation of cellular retinoic acid-binding protein 1, GPR50 and nestin in tanycytes of the third ventricle ependymal layer of the Siberian hamster. J Endocrinol. 2006;191:687-698.

20. Knowles F, Kumar TCA. Structural changes, related to reproduction, in the hypothalamus and in the pars tuberalis of the rhesus monkey. Philos Trans R Soc Lond B Biol Sci. 1969;256:357-375.

21. Symington RB, Hayes MM, Knight BK, Grizic A. Histological studies of the ependymal cells of the human third ventricle. S Afr Med J. 1973;47:2273-2278.

22. Nett TM, Adams TE. Further studies on the radioimmunoassay of gonadotropin-releasing hormone: effect of radioiodination, antiserum and unextracted serum on levels of immunoreactivity in serum. Endocrinology. 1977;101:1135-1144.

23. Gill S, Sharpless JL, Rado K, Hall JE. Evidence that GnRH decreases with gonadal postmenopausal women. J Clin Endocrinol Metab. 2002;87:2290-2296.

24. Hall JE. Neuroendocrine physiology of the early and late menopause. Endocrinol Metab Clin North Am. 2004;33:637-659.

25. Rance NE, Uswandi SV. Gonadotropin-releasing hormone gene expression is increased in the medial basal hypothalamus of postmenopausal women. J Clin Endocrinol Metab. 1996;81:3540-3546.

26. Porchet R, Probst A, Bouras C, Dráberová E, Dráber P, Riederer BM. Analysis of glial acidic fibrillary protein in the human entorhinal cortex during aging and in Alzheimer's disease. Proteomics. 2003;3:1476-1485.

27. Salminen A, Ojala J, Kaarniranta K, Haapasalo A, Hiltunen M, Soininen $\mathrm{H}$. Astrocytes in the aging brain express characteristics of senescenceassociated secretory phenotype. Eur J Neurosci. 2011;34:3-11.

28. Koutcherov Y, Mai JK, Ashwell KWS, Paxinos G. Organization of human hypothalamus in fetal development. J Comp Neurol. 2002;446:301-324.

29. Kuiri-Hänninen T, Sankilampi U, Dunkel L. Activation of the hypothalamic-pituitary-gonadal axis in infancy: minipuberty. Horm Res Paediatr. 2014;82:73-80.

30. Ojeda SR, Lomniczi A, Mastronardi C, et al. Minireview: the neuroendocrine regulation of puberty: is the time ripe for a systems biology approach? Endocrinology. 2006;147:1166-1174.

31. Prevot V, Cornea A, Mungenast A, Smiley G, Ojeda SR. Activation of erbB-1 signaling in tanycytes of the median eminence stimulates transforming growth factor $\beta 1$ release via prostaglandin E2 production and induces cell plasticity. J Neurosci. 2003;23:10622-10632.

32. Ojeda SR, Prevot V, Heger S, Lomniczi A, Dziedzic B, Mungenast A. Glia-to-neuron signaling and the neuroendocrine control of female puberty. Ann Med. 2003;35:244-255.

33. Bruni JE, Clattenburg RE, Millar E. Tanycyte ependymal cells in the third ventricle of young and adult rats: a Golgi study. Anat Anz. 1983;153:53-68.

34. Robins SC, Stewart I, McNay DE, et al. $\alpha$-Tanycytes of the adult hypothalamic third ventricle include distinct populations of FGFresponsive neural progenitors. Nat Commun. 2013;4:679-683.

35. Bolborea M, Dale N. Hypothalamic tanycytes: potential roles in the control of feeding and energy balance. Trends Neurosci. 2013:36:91-100

36. Goodman T, Hajihosseini MK. Hypothalamic tanycytes - masters and servants of metabolic, neuroendocrine, and neurogenic functions. Front Neurosci. 2015;9:387.

How to cite this article: Koopman ACM, Taziaux M, Bakker J. Age-related changes in the morphology of tanycytes in the human female infundibular nucleus/median eminence. J Neuroendocrinol. 2017;29:1-9. https://doi.org/10.1111/jne.12467 